\title{
Cardiac Arrest
}

National Cancer Institute

\section{Source}

National Cancer Institute. Cardiac Arrest. NCI Thesaurus. Code C50479.

The sudden cessation of cardiac activity in an individual who becomes unresponsive,

without normal breathing and no signs of circulation. Cardiac arrest may be reversed by CPR, and/or defibrillation, cardioversion or cardiac pacing. 